Article

\title{
Performance Degradation of silicon based magnetorheological elastomer under accelerated weathering treatment
}

\author{
W. Wibowo ${ }^{1}$, Bhre Wangsa Lenggana ${ }^{1}$, U. Ubaidillah ${ }^{1}{ }^{*}$, Dody Ariawan ${ }^{1}$, Fitrian Imaduddin ${ }^{1}$ and \\ Saiful Amri Mazlan ${ }^{*}$ \\ ${ }^{1}$ Mechanical Engineering Department, Universitas Sebelas Maret, Jl. ISurakarta, Indonesia \\ 2 Engineering Materials and Structures (eMast) iKohza, Malaysia-Japan International Institute of \\ Technology(MJIIT), Universiti Teknologi Malaysia, Jalan Sultan Yahya Petra, 54100, Kuala Lumpur, \\ Malaysia \\ *Correspondence: ubaidillah_ft@staff.uns.ac.id; amri.kl@utm.my
}

\begin{abstract}
Silicone RTV-based engineering rubber composite products have been widely used for several applications in various fields as a major component such as structure, automotive, and medical. In its application, the rubber composite product is placed in an open area that is directly exposed to sunlight and rain. It has a significant negative impact on changes in chemical and rheological properties, making the product life of rubber composite products shorter. Therefore, in this study, changes in the chemical and rheological properties of both isotropic and anisotropic magnetorheological elastomer (MRE) treated with accelerated weathering were studied compared to untreated specimens with specimens that had been treated. MRE specimens with $40 \%$ by weight CIP were prepared with no current excitation and another sample were made under $1.5 \mathrm{~T}$ of magnetic flux density. Each specimen was treated in an accelerated weathering machine Q-Sun Xe1 Xenon Test Chamber with a UV light exposure cycle for 102 minutes and 18 minutes of UV light combined with water spray for 24 hours followed by a condensation cycle of 4 hours in a dark period. Material characterization was carried out using FTIR and Rheometer to determine the changes in chemical and rheological properties. The morphological analysis results showed that the surface was rough and more cavities occurred after being given weather treatment. Rheometer test results showed a decrease in storage modulus in each MRE specimen that had been treated compared to untreated MRE specimens. Meanwhile, FTIR testing showed a change in wave peak between untreated and treated MRE specimens. Based on overal results, the artificial weather was proven to be giving serious impact on silicon RTV based MRE. This finding might be used as quantitative consideration for whom designing semi-active devices based on silicon RTV matrix.
\end{abstract}

Keywords: magnetorheological, elastomer, magnetorheological elastomer, MRE, weather, accelerated, rubber, composite, rheological

\section{Introduction}

In its development, rubber products have progressed quite rapidly. One of them is an engineered rubber product which has now been widely applied in various engineering fields. This is because engineered rubber products have excellent physical properties and durability as well as their ability to deform strength. Rubber composites are one of the engineered rubber products that are widely applied in various fields such as marine [1], structural, automotive [2, 3], aerospace [4]. Several studies have shown that rubber composite products have vital environmental sensitivity compared to metal structures, especially in their space sector application. However, on the other hand, engineered rubber products such as composites are very susceptible to the conditions of ambient temperature, humidity, heat, and chemicals that hit them. In general, the aging of rubber composites is caused by chemicals [5, 6], thermal, heat [7, 8], oxidative destructive [9-11], and moisture [12]. These 
conditions are conditions that depend on the weather. Thus, the weather conditions around rubber composite products' application negatively affect their physical properties [13], especially the degradation or aging stability of rubber composites. In rubber composites, the aging phenomenon is an irreversible process and can significantly change its physical, mechanical, and rheological properties $[13,14]$. The change in interface adhesion in reinforcement and rubber causes hardening of the rubber composite, resulting in embrittlement and reduced rubber life.

In addition to general rubber products applied in the automotive sector, such as tires or other specialty rubber products, rubber composites in the form of sensors, magnetic and electromagnetic field protection materials have been proposed in recent decades. For this kind of requirement, a new type of rubber composite with non-conventional filler as an integrated active component is required [15]. One way that this can be done for this purpose is to prepare a new material with a specific filler such as magnetic particles. Magnetorheological elastomers (MRE) are the new generation materials that have been widely explored for their excellent flexibility, easy to form, and good sensitivity to magnetic fields $[15,16]$. However, despite its excellent capabilities, MRE as one of the composite materials is still very vulnerable to damage due to the influence from the environment or the surrounding weather [17]. It is generally recognized that the mechanical properties and durability of MRE are greatly affected by the MRE aging, resulting in damage. One example is the study conducted by Kruzelak et al [10], which showed an increase in the cross-link density of magnetic composites due to the formation of rubber chain oxidation during oxidative aging. His observations on the mechanical properties associated with the rubber magnetic composites' modulus and hardness were increased compared to the samples without aging. Thermo-oxidative aging has also been studied in MRE samples by Masbowski et al [18]. On MRE samples with fillers of carbonyl iron particles (CIP) and carbon black which were treated by being put in a drying oven at $70^{\circ} \mathrm{C}$ for two weeks [19]. The result was that the mechanical properties of MRE slightly deteriorated due to the oxidative aging process. Other researchers made similar conclusions that tensile strength, tear strength, and hardness decreased significantly up to $72 \%$ due to an increase in temperature and aging time. This is due to molecular configuration changes accompanied by hydrothermal deterioration that causes micro-gaps to develop from the matrix and the fill/matrix interface.

Treatment related to MRE degredation has been carried out in several forms such as thermal treatment, but some of these treatments cannot represent weather behavior. It is well known that environmental impact during application of MRE based devices cannot be represented by thermal only. Moreover, based on previous studies, the rubber composite/MRE studies were limited to specific rubber matrices and mainly focused on mechanical properties and cross-linking [20]. Intensive studies on MRE resistance based on silicon RTV matrices to natural conditions such as sunlight and heat, and rainfall have not received attention [21, 22]. Meanwhile, the application of rubber composite products such as MRE is mostly made in open environments that are directly exposed to sunlight and rain [23,24]. Therefore, this article contributes to the effect of weathering using an accelerated weathering machine on MRE specimens based on silicon RTV, both isotropic and anisotropic types. The novel finding would be realized by examining the rheological properties and material deterioration under infrared testing. The main finding could be important for researchers or designer which will decide silicon as the MRE matrix.

\section{Materials and Methods}

\subsection{Samples Preparation}

In this study, MRE based on silicone RTV consisted of $40 \% \mathrm{wt} \%$ CIPs and silicon RTV as a suspending medium was prepared. The preparation sample of MRE is shown in Figure 2.1. 


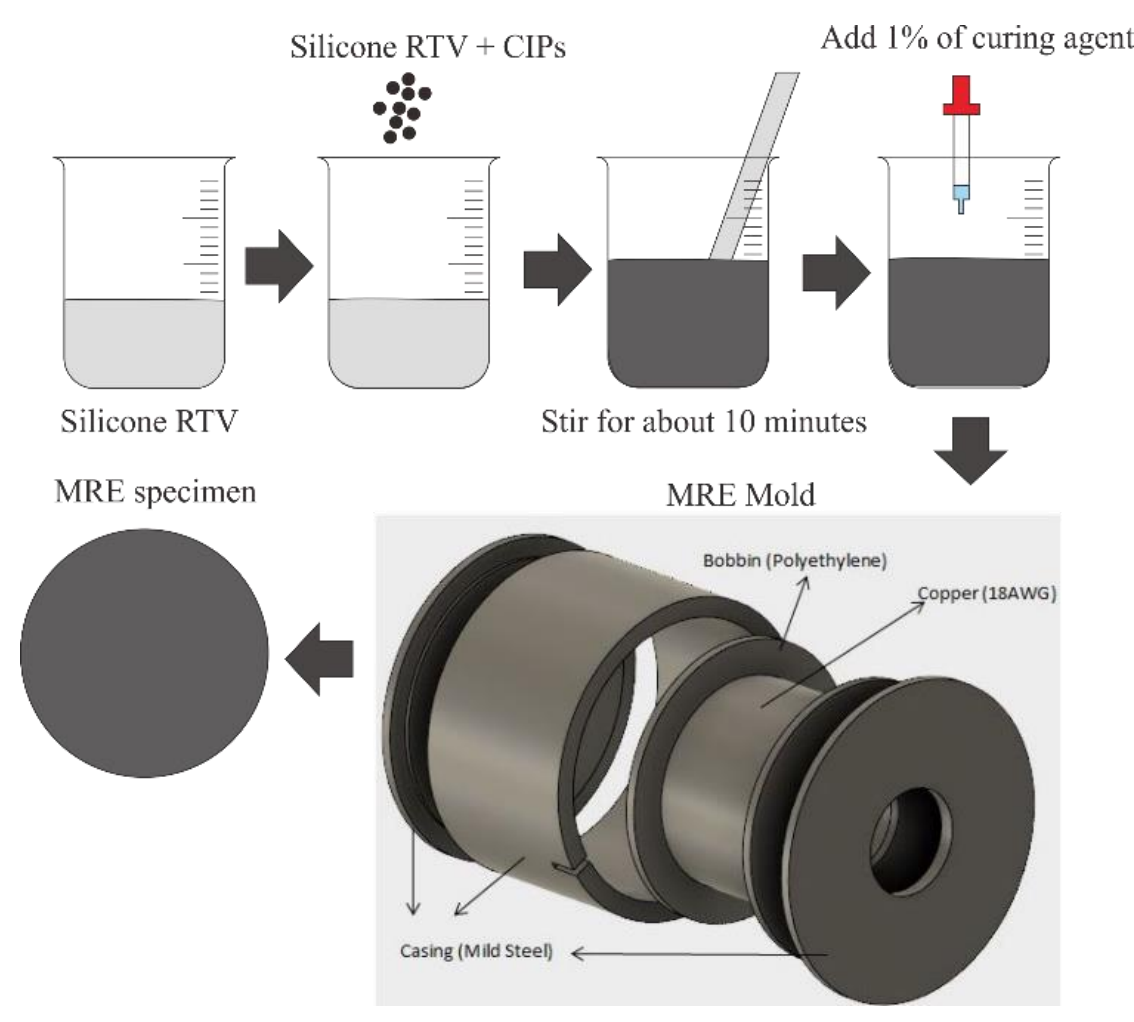

Figure 2.1 MRE sample preparation

The samples were made in both isotropic and anisotropic types using the same equipment. For the anisotropic MRE samples, the mixed silicon RTV and CIP were cured under the influence of an magnetic flux density of $0.5 \mathrm{~T}$. This flux magnetic value was obtained by applying $1 \mathrm{~A}$ DC current. To convice the value, before the molding was filled with the mixture, the flux density was measured using gauss meter. The MRE sample was stirred conventionally for about 10 minutes at room temperature $\left(25^{\circ} \mathrm{C}\right)$ until it looked homogenous. Then, $1 \%$ curing agent, NS 625 B (Nippon Steel) was added to the MRE sample that had been stirred before pouring it into the mold. To remove small bubbles, the mixtures were palced in vacuum chamber fo degassing purposes [15].

\subsection{Sample Characterization}

The test method followed the standard test method for rubber deterioration using artificial weathering apparatus. This method was conducted in an accelerated weathering machine Q-SUN Xe-1 Xenon Test Chamber and ASTM D750. The Q-SUN Xe-1 Xenon Test Chamber is a tabletop chamber that reproduces the damage caused by full-spectrum UV light using a single xenon arc lamp with optional water spray and chiller.

As mentioned in ASTM D750, the specimens should have a maximum thickness of $0.75 \mathrm{~mm}$ and a minimum thickness of $0.60 \mathrm{~mm}(0.025 \mathrm{in})$, and the wide of specimens are not critical. Along with the ASTM standard thickness, the samples with thickness of $1 \mathrm{~mm}$ were also treated for the needs of rheology testing which requires minimum thickness of $1 \mathrm{~mm}$. The default exposure shall be $102 \mathrm{~min}$ light only followed by $18 \mathrm{~min}$ light plus either water spray on the front surface or immersion in water. The black panel temperature (BPT) for this test is using $63^{\circ} \mathrm{C}$ during the dry period of exposure to light with relative humidity $60 \%$ during this exposure. The irradiance level shall be controlled at 340 $\mathrm{nm}$. This test shall be running with the cycle in 8 hours of UV exposure in an un-insulated black panel with a temperature of $60^{\circ} \mathrm{C}$ and followed by condensation cycle in 4 hours dark period with wetting un-insulated black panel temperature of $50^{\circ} \mathrm{C}$. The samples obtained were MRE samples that had been treated and without treatment for both isotropic and anisotropic samples.

The prepared sample was then characterized under several testing such as morphology, rheology and fourier transform infrared (FTIR). To check the surface of treated and untreated 
samples, image capture for micrograph analysis using a euromex microscope model "F" range, Holland was performed at ten magnification (M10). This enlargement is clear enough to see the difference between the treated and untreated samples. Micrograph analysis is carried out on the surface of the sample, because during the treatment with an accelerated weathering machine the surface of the sample is directly exposed to both UV rays and water. The MRE sample for rheometer testing was cut to a diameter of $20 \mathrm{~mm}$. This test was performed using a Rheometer MCR 302 Anton Paar Companies machine. The amount of the MRE sample between CIPs and silicon rubber medium in this study was based on the weight ratio. The two ingredients were stirred manually at room temperature for 10 minutes until homogeneous. After the homogeneous, sample was poured on the molding and leveled before clotting. The sample was tested with a rheometer at $25^{\circ} \mathrm{C}$. In addition to rheometer testing, FTIR testing is also carried out on samples to determine changes or differences in chemical compound between untreated and treated MRE samples. The MRE samples to be tested by FTIR were crushed until smooth using a mortar stamper and they were mixed thoroughly with kalium bromide $(\mathrm{KBr})$. The FTIR testing used Shimadzu Prestige $21 \mathrm{IR}$, Japan with a wavelength specification of $12500-240 \mathrm{~cm}^{-1}$ and an accuracy level of $\pm 0.125 \mathrm{~cm}^{-1}$. Spectrum resolution on this machine ranges from $0.5-2 \mathrm{~cm}^{-1}$.

\section{Results and Discussion}

\subsection{Micrograph Analysis}

Micrograph studies on the surface of treated and untreated MRE samples are essential for analyzing the degree of degradation. Figure 3.1 (a) shows an untreated micrograph sample, and Figure 3.1 (b) shows a micrograph sample with weather treatment using an accelerated weathering machine. The effects of surface degradation can be observed from these images [25, 26]. The results show an apparent difference.

After treatment of the MRE sample, the surface of the sample was found to be more irregular than that of the virgin sample.

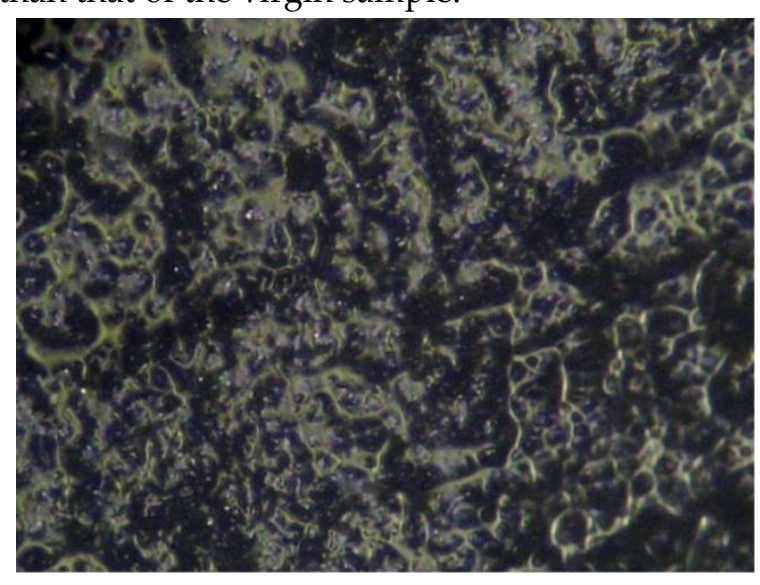

(a)

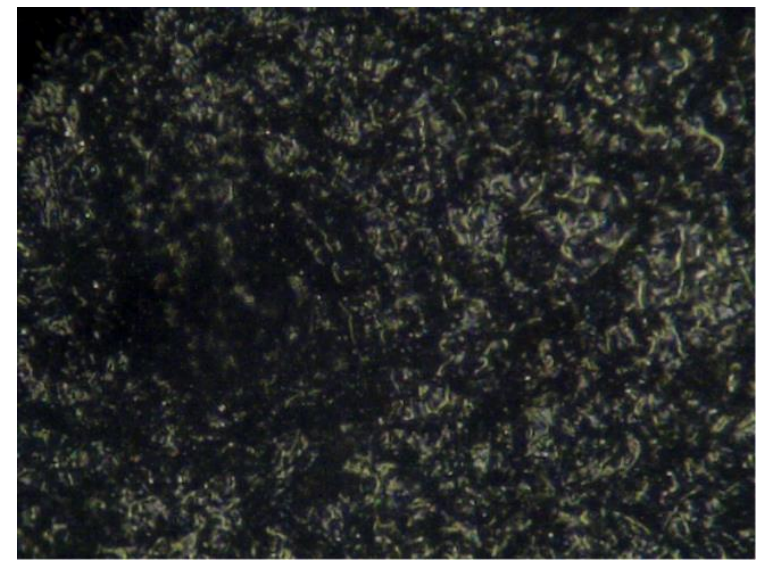

(b)

Figure 3.1 Micrograph analysis of anisotropic MRE (a) untreated (1.5 T) and (b) treated (1.5 T)

On the other hand, the change in roughness of the treated samples looks obviously, even using a regular microscope. Surface defects is possibly due to the evolution of low molecular degradation products, allow the penetration of water and possible decomposing agents (e.g, enzymes) into the bulk of the polymer and facilitate further environmental degradation. This phenomenon was also found by other researcher which tested MRE with thermal aging [27].

\subsection{Rheological Properties}

The storage modulus of all samples with and without weather treatment is depicted in Figure 3.2. As shown in Figure 3.2 it is clear that the storage modulus at each MRE sample flow strength, 
both anisotropic and isotropic, has decreased. In Figure 3.2 (c) the treated anisotropic MRE sample shows a storage modulus value that is almost the same as the storage modulus of an isotropic MRE sample without treatment. This indicates that weather treatment can damage the particle chains' arrangement that occurs in anisotropic MRE samples [28-31]. The breakdown of the particle chain arrangement occurred in all anisotropic MRE specimens and brought all storage modulus values close to the storage modulus of the isotropic MRE samples.

Figure 3.2 shows that the anisotropic MRE sample without treatment has a peak storage modulus value of $1.9 \mathrm{MPa}$ at the current input of $4 \mathrm{~A}$. Simultaneously, the peak storage modulus value of the anisotropic MRE sample treated at the same current input (4 A) is $0.37 \mathrm{MPa}$.

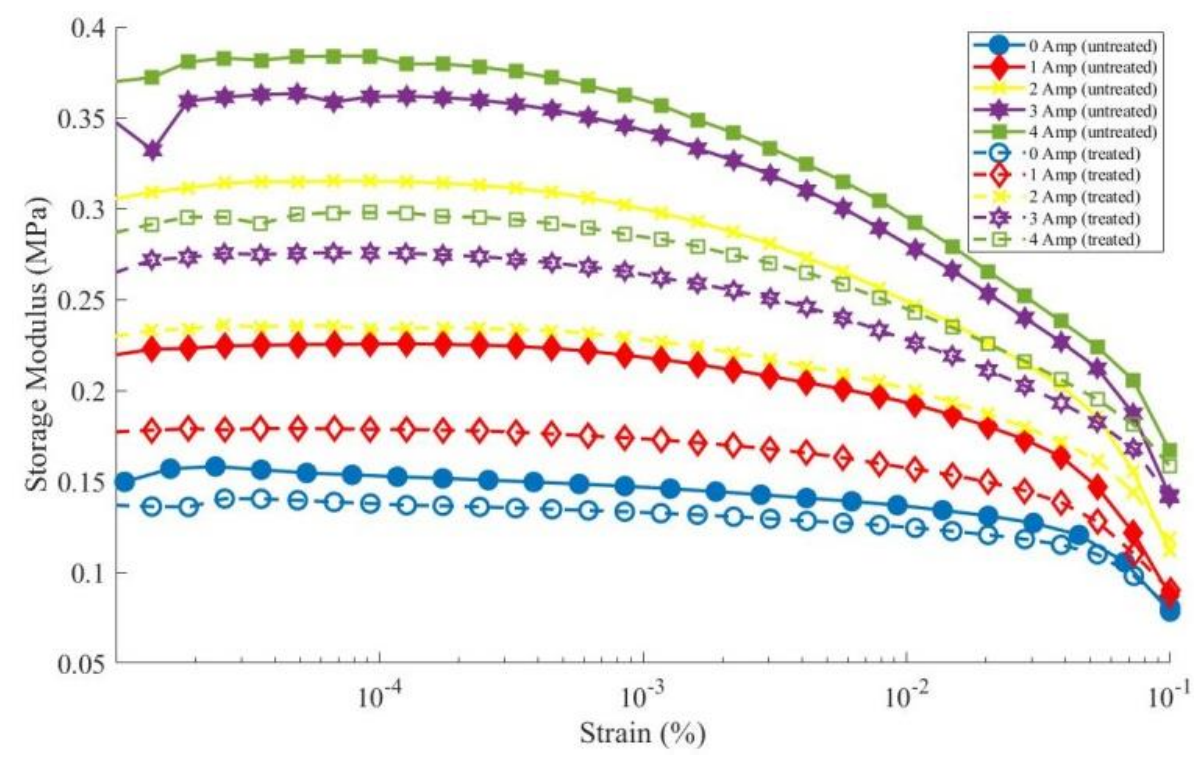

(a)

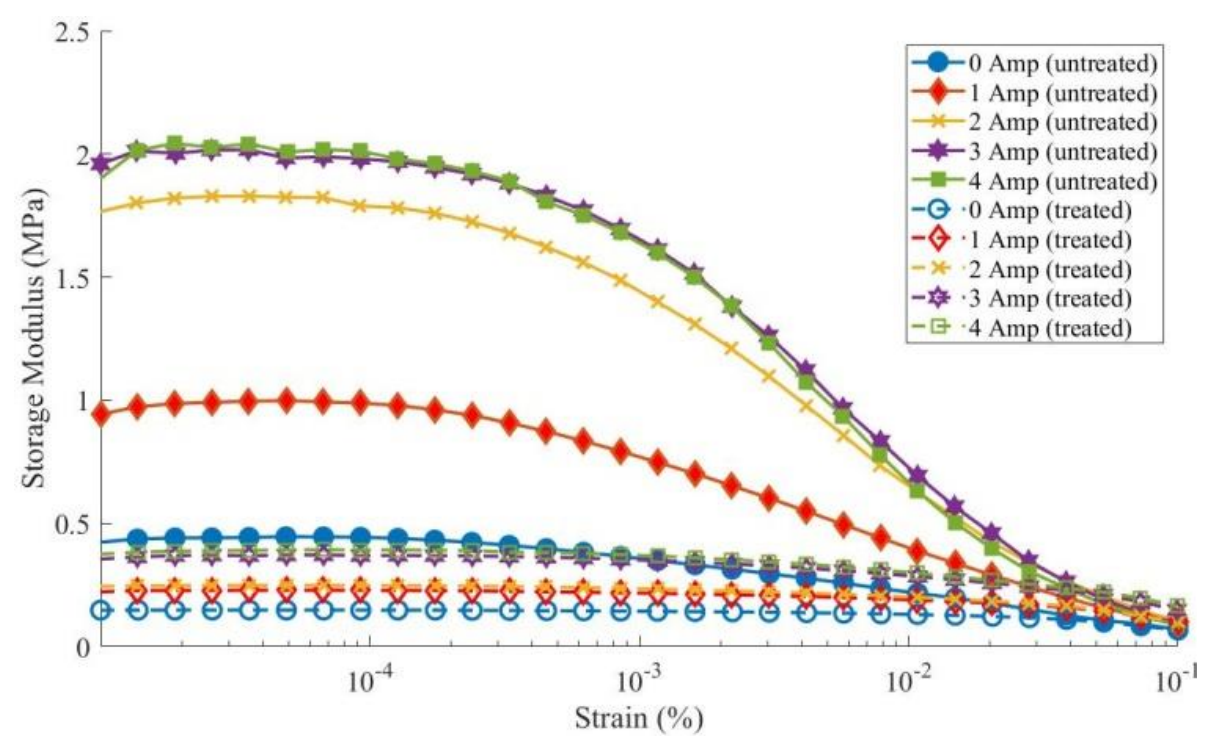

(b) 


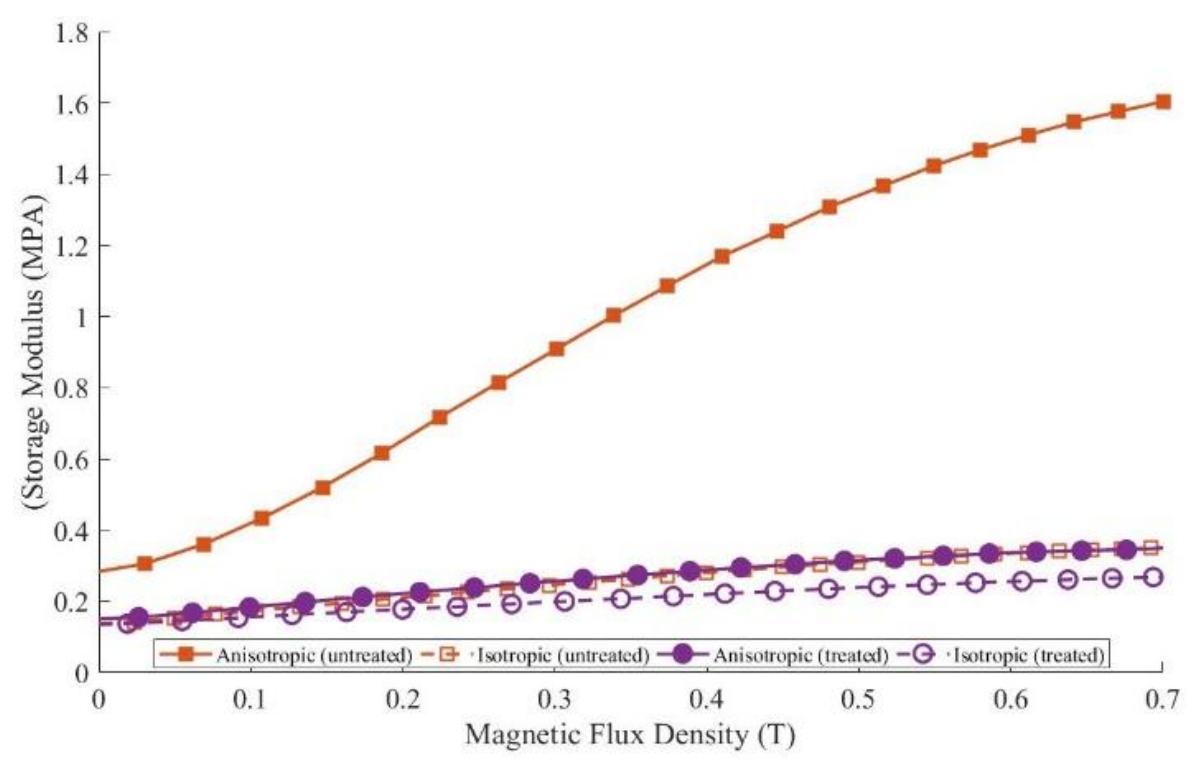

(c)

Figure 3.2 Result of rheometer characterization (a) anisotropic; (b) isotropic; and (c) comparison of anisotropic and isotropic under ramp frequency test

This shows that the storage modulus value has decreased by $80.5 \%$ due to the influence of weather (exposure to UV rays and water spraying), which is carried out in a cycle. A decrease in the storage modulus value occurred in all current input in the rheometer test. The decrease in the storage modulus value with currents of $0,1,2$, and 3 Amperes was $66.67 \%, 78.9 \%, 85.2 \%$, and $80.5 \%$, respectively. This reduction is very significant compared to the isotropic MRE sample, as shown in Figure 3.3. This is because the anisotropic MRE sample has a high storage modulus value due to the arrangement of the particle chains formed due to the input of electric current given during the curing process of making MRE samples.

Figure 3.3 shows the results of the isotropic MRE sample rheometer test treated and untreated. The peak value of storage modulus to isotropic MRE without treatment only reaches $0.37 \mathrm{MPa}$ at $4 \mathrm{~A}$ current input when testing. This value is almost the same as the storage modulus value of the anisotropic MRE sample treated. Meanwhile, the isotropic treated MRE sample's storage modulus value only reached a peak value of $0.28 \mathrm{MPa}$. This shows a decrease in the value of the isotropic MRE sample's storage modulus at the 4 A current input is $24.3 \%$. This reduction value is not comparable to the anisotropic MRE sample, which achieved a mean reduction of up to $78.3 \%$. This occurs because the arrangement of the particle chains in the isotropic MRE sample is irregular, so that the weather treatment given to the sample does not damage the arrangement but only makes its rheological properties degraded $[32,33]$. 


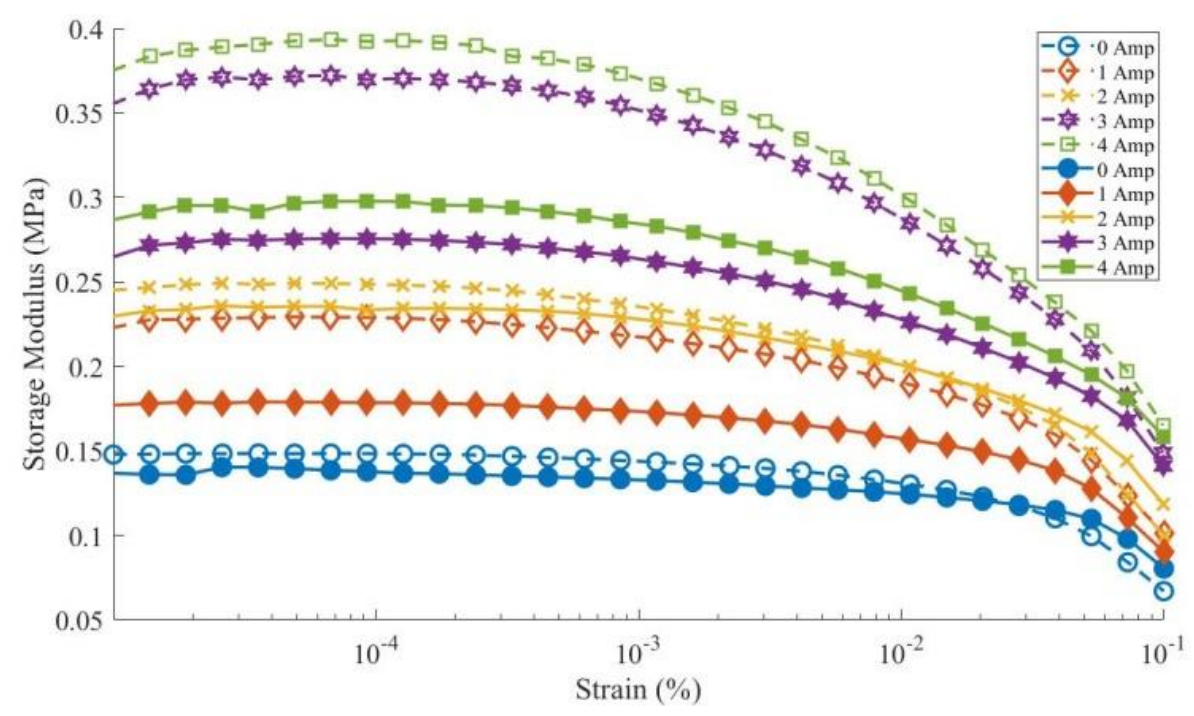

Figure 3.3 Results of the isotropic MRE sample rheometer test for treated and untreated

\subsection{FTIR Testing}

FTIR testing that has been carried out on the test samples produces data in the form of a graph of wavelength $\left(\mathrm{cm}^{1}\right)$ vs \% Transmission, as shown in Figure 3.4. The graph shows the existence of a transmission in the form of a peak to be interpreted. The interpretation results show the bonds formed in the test specimens due to weathering treatment with the accelerated weathering machine and the specimens without treatment.

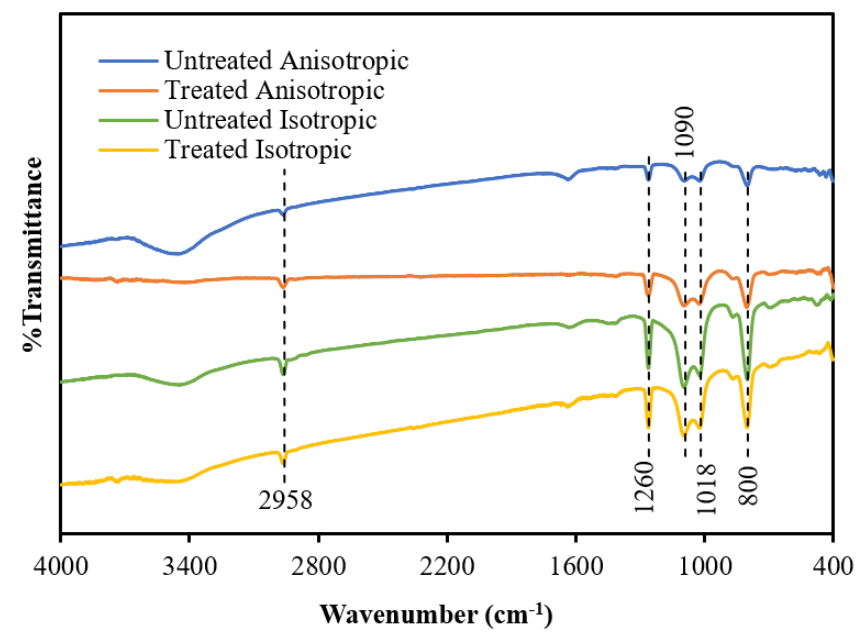

Fig. 3.4 FTIR result characterization

The functional groups of the sample were elucidated with Fourier Transformation Infrared (FTIR) spectroscopy and the result is presented in Figure 3.4. The peak at around 2958 and $1260 \mathrm{~cm}-$ 1 attributed to stretching and bending vibration of Si-(CH3)2 [34]. Meanwhile, two absorption band at 1090 and $1018 \mathrm{~cm}-1$ assigned to characteristic of silicone rubber for -Si-CH2-CH3 [35]. Then, the peak at around $800 \mathrm{~cm}-1$ corresponds to silanol groups that could be deconvoluted to three peaks as illustrated in Figure 3.6 using equation 1. The peaks at 795, 800 and 810 are Si-O-Si, terminal Si-OH and unbonded $\mathrm{Si}-\mathrm{O}$ [36]. FTIR deconvolution is a method to estimate quantitative interaction in a certain functional group [37]. The intensity of the bond formation in each specimen looks the same. However, there is a difference at a wavelength of $3451 \mathrm{~cm}-1$, the untreated specimens show the formation of a peak with a reasonably comprehensive intensity, compared to specimens treated both for anisotropic specimens and isotropic specimens. There was almost no peak appearance in the 
treated specimens, as shown in the results of the treated anisotropic specimens. This indicates that the $\mathrm{OH}$ group formed before the specimen was treated faded due to the influence of UV exposure.

Of all tested MRE specimens, no absorption was found at 560-570 cm- 1 which should be the iron oxide or Fe-O absorption region [38, 39]. This behavior is called a Binder bond [40]. The binder bond occurs due to the neutralization of the carbonyl charge so that it cannot interact with infrared wavelengths. Then, by using deconvolution method, the Gaussian peak area is presented in Figure 3.5 The peak area of treated sample shows decreasing trends may be due to detaching of the iron filler from silicon rubber. Finally, it is also evidenced that the iron carbonyl as a filler has no chemical interaction with silicon rubber as a result of rubber coated the iron filler as also reported in another reference [41].

$$
\mathrm{Y}=\frac{A}{w} \sqrt{\frac{4 \ln 2}{\pi}} \frac{1}{4 \ln 2} \frac{(x-x c)^{2}}{w^{2}}
$$

Where A and $\mathrm{w}$ are area and fullwidth of the graph, respectively with $\mathrm{xc}$ as the center.

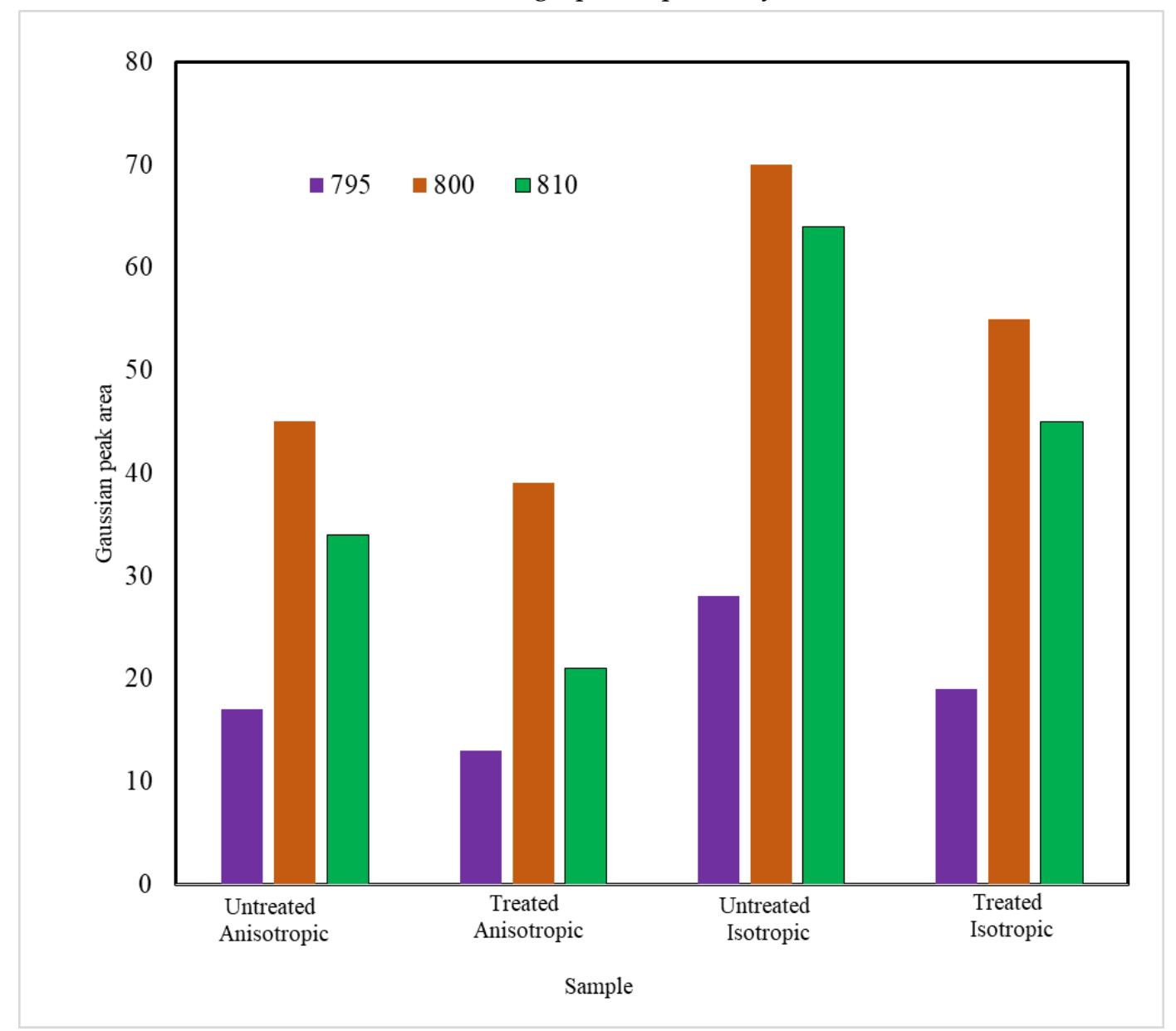

Figure 3.5 Gaussian peak area at $800 \mathrm{~cm}-1$ 

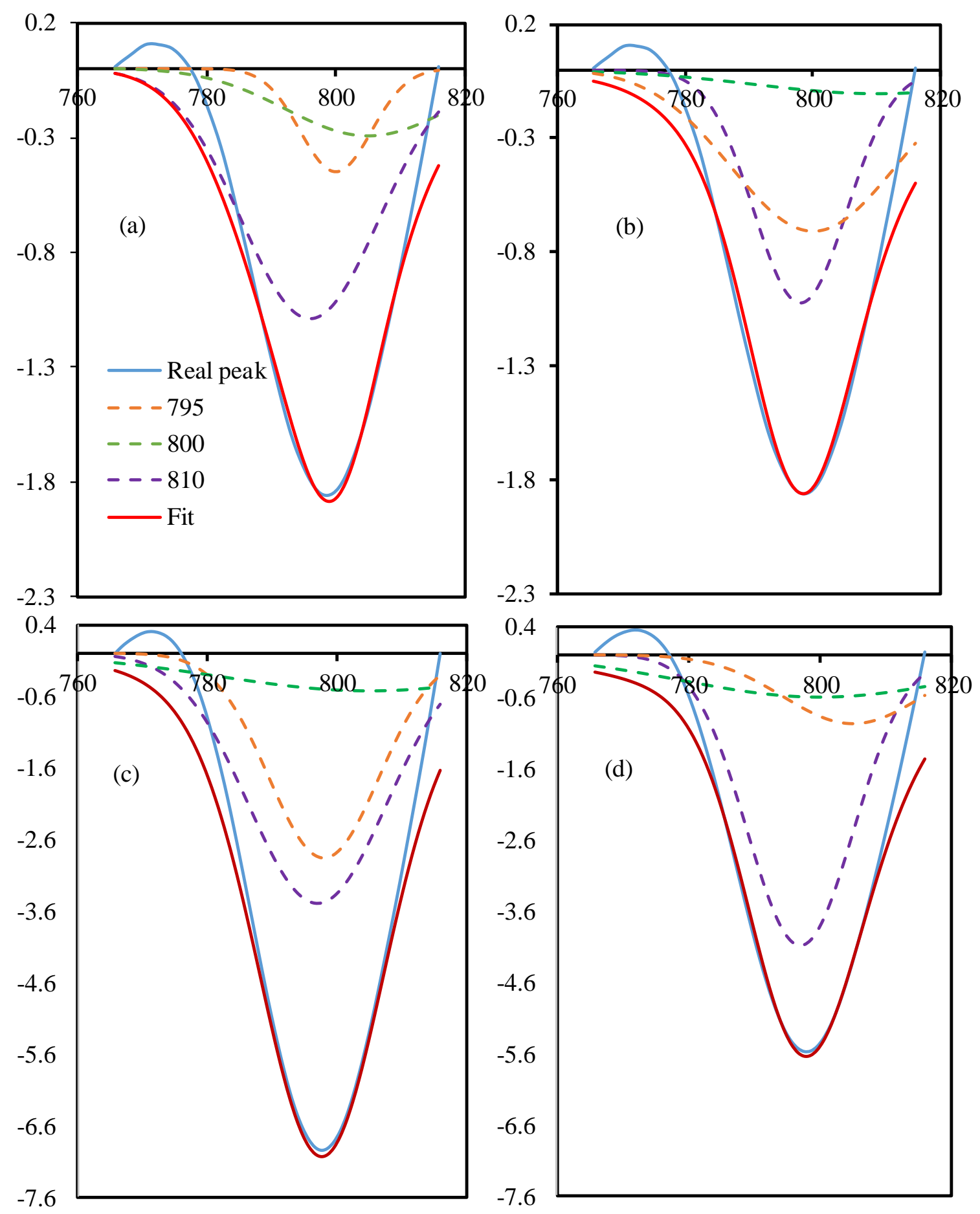

Figure 3.6 FTIR deconvolution of peak $800 \mathrm{~cm}-1$

\section{Conclusions}

MRE sample characterization of both treated and untreated has been carried out to determine the quantitative impact of artificial environment on their performance degradation. The characterization test results with the analysis micrograph showed a significant difference between the samples given treatment and the samples without treatment. Degradation of the treated sample is evident in the samples increased roughness and the sample particle arrangement's irregularity on the surface. The results of the characterization test using rheology showed a very significant reduction in storage modulus. This decrease occurred up to $80.5 \%$. This occurs due to the aging of the samples treated using an accelerated weathering machine. Exposure to UV light and continuous 
and repeated spraying of water on the sample is indicated to accelerate the sample aging process. This process occurs by breaking the chain of particle bonds arranged in the MRE sample so that the MRE particle chain formed in the anisotropic sample is damaged and becomes like an isotropic sample. In FTIR testing, each sample does not have much difference. All samples show the appearance of a peak at the same wavelength with almost the same intensity. Nevertheless, what distinguishes it is at a wavelength of $3451 \mathrm{~cm}-1$, the intensity of the untreated sample in forming $\mathrm{OH}$ groups is very low, even in anisotropic samples, it hardly forms a peak at that wavelength. Thus, based on the results of the MRE characterization by being given weather treatment using an accelerated weathering machine and without treatment, the samples' aging was faster so that a decrease in sample performance occurs. Exposure to UV light and spraying water greatly affected MRE's performance, which is widely applied in open environments. This decrease in performance was evident in the rheological characterization test, which showed that the sample's storage modulus decreased significantly compared to untreated virgin samples. It is a new challenge for developing research in the field of advanced materials, magnetorheological elastomers in particular.

\section{Patents}

No patent resulted from this manuscript.

Supplementary Materials: Supplementary materials can be found at www.mdpi.com/xxx/s1.

Author Contributions: For research articles with several authors, a short paragraph specifying their individual contributions must be provided. The following statements should be used "Conceptualization, W. and U.U.; methodology, W.; software, B.W.L.; validation, W., S.A.M. and U.U.; formal analysis, W.; investigation, W.; resources, W.; data curation, W., S.A.M.; writing - original draft preparation, W.; writing-review and editing, W., B.W, U.U.; visualization, B.W.L.; supervision, D.A., F.I, U.U..; project administration, W., U.U..; funding acquisition, U.U.. All authors have read and agreed to the published version of the manuscript.", please turn to the CRediT taxonomy for the term explanation. Authorship must be limited to those who have contributed substantially to the work reported.

Funding: Please add: This research was funded by Universitas Sebelas Maret, grant number Hibah Doktor 2021" and "The APC was funded by Universitas Sebelas Maret" under scheme Insentif Bantuan Publikasi 2021.

Acknowledgments: Authors thank to Dr. Siti Aishah Abd. Aziz which help setting up rheological measurement in G3 Lab, UTM Kuala Lumpur. We also thank to Mr. Ahmad Mas'udi for the useful discussion on FTIR analysis.

Conflicts of Interest: The authors declare no conflict of interest."

\section{Abbreviations}

MRE Magnetorheological elastomers

FTIR Fourier Transform Infra Red

\section{Appendix A}

The appendix is an optional section that can contain details and data supplemental to the main text. For example, explanations of experimental details that would disrupt the flow of the main text, but nonetheless remain crucial to understanding and reproducing the research shown; figures of replicates for experiments of which representative data is shown in the main text can be added here if brief, or as Supplementary data. Mathematical proofs of results not central to the paper can be added as an appendix.

\section{Appendix B}

All appendix sections must be cited in the main text. In the appendixes, Figures, Tables, etc. should be labeled starting with 'A', e.g., Figure A1, Figure A2, etc. 


\section{References}

References must be numbered in order of appearance in the text (including citations in tables and legends) and listed individually at the end of the manuscript. We recommend preparing the references with a bibliography software package, such as EndNote, ReferenceManager or Zotero to avoid typing mistakes and duplicated references. Include the digital object identifier (DOI) for all references where available.

Citations and References in Supplementary files are permitted provided that they also appear in the reference list here.

In the text, reference numbers should be placed in square brackets [ ], and placed before the punctuation; for example [1], [1-3] or [1,3]. For embedded citations in the text with pagination, use both parentheses and brackets to indicate the reference number and page numbers; for example [5] (p. 10), or [6] (pp. 101-105).

1. Bus, T.; Dale, M. L.; Reynolds. K. J.; Bastiaansen, C. W. M. Thermoplastic, rubber-like marine antifouling coatings with micro-structures via mechanical embossing, Biofouling, 2020, 36, 138-145. https://doi.org/10.1080/08927014.2020.1734576.

2. Louda, P.; Applications of thin coatings in automotive industry, J. Achiev. Mater. Manuf. Eng.; 2007. 24, 51-56. http://jamme.acmsse.h2.pl/papers_vol24_1/24105.pdf.

3. Fentahun, M. A.; Savas, M. A.; Materials Used in Automotive Manufacture and Material Selection Using Ashby Charts, Int. J. Mater. Eng.; 2018, 8, 40-54. https://doi.org/10.5923/j.ijme.20180803.02.

4. Zhang, H.; Xu, G.; Chen, X.; Wang, R.; Shen, K.; Effect of long-term laboratory aging on rheological properties and cracking resistance of polymer-modified asphalt binders at intermediate and low temperature range, Constr. Build. Mater.; 2019, 226, 767-777. https://doi.org/10.1016/j.conbuildmat.2019.07.206.

5. García-Moreno, I.; Caminero, M.; Rodríguez, G.; López-Cela, J.; Effect of Thermal Ageing on the Impact and Flexural Damage Behaviour of Carbon Fibre-Reinforced Epoxy Laminates, Polymers (Basel), 2019, 11, 80. https://doi.org/10.3390/polym11010080.

6. Kashi, S.; De Souza, M.; Al-Assafi, S.; Varley, R.; Understanding the Effects of In-Service Temperature and Functional Fluid on the Ageing of Silicone Rubber, Polymers (Basel), 2019, 11, 388. https://doi.org/10.3390/polym11030388.

7. Kashi, S.; Varley, R.; De Souza, M.; Al-Assafi, S.; Di Pietro, A.; de Lavigne, C.; Fox, B.; Mechanical, Thermal, and Morphological Behavior of Silicone Rubber during Accelerated Aging, Polym. Plast. Technol. Eng.; 2018, 57, 1687-1696. https://doi.org/10.1080/03602559.2017.1419487.

8. Ahmed, K.; Sirajuddin Nizami, S.; Zahid Raza, N.; Shirin, K.; Cure Characteristics, Mechanical and Swelling Properties of Marble Sludge Filled EPDM Modified Chloroprene Rubber Blends, Adv. Mater. Phys. Chem.; 2012, 02, 90-97. https://doi.org/10.4236/ampc.2012.22016.

9. Lokander, M.; Reitberger, T.; Stenberg, B.; Oxidation of natural rubber-based magnetorheological elastomers, Polym. Degrad. Stab.; 2004, 86, 467-471. https://doi.org/10.1016/j.polymdegradstab.2004.05.019.

10. Kruželák, J.; Hudec, I.; Dosoudil, R.; Influence of thermo-oxidative and ozone ageing on the properties of elastomeric magnetic composites, Polym. Degrad. Stab.; 2012, 97, 921-928. https://doi.org/10.1016/j.polymdegradstab.2012.03.025.

11. Zhao, G.; Shi, L.; Zhang, D.; Feng, X.; Yuan, S.; Zhuo, J.; Synergistic effect of nanobarite and carbon black fillers in natural rubber matrix, Mater. Des.; 2012, 35, 847-853. https://doi.org/10.1016/j.matdes.2011.05.056.

12. Salim, M. S.; Ariawan, D.; Rasyid, M. F. A.; Taib, R. M.; Thirmizir, M. Z. A.; Ishak, Z. A. M.; Accelerated weathering and water absorption behavior of kenaf fiber reinforced acrylic based polyester composites, Front. Mater.; 2020, 7. doi: 10.3389/fmats.2020.00026

13. Tang, N.; Lv, Q.; Huang, W.; Lin, P.; Yan, C.; Chemical and rheological evaluation of aging characteristics of terminal blend rubberized asphalt binder, Constr. Build. Mater., 2019, 205, 87-96. https://doi.org/10.1016/j.conbuildmat.2019.02.008.

14. Verma, A. R. and Reddy, B. S.; Accelerated Ageing Studies of Silicon-Rubber based Polymeric Insulators Used for HV Transmission Lines Science direct. Polym. Test.; 2017, 62, 124-131. 
15. Aziz, S. A. A.; Mazlan, S. A.; Ismail, N. I. N.; Ubaidillah, Choi, S. B.; Nordin, N. A.; Mohamad, N.; A comparative assessment of different dispersing aids in enhancing magnetorheological elastomer properties, Smart Mater. Struct. 2018, 27, 117002. https://doi.org/10.1088/1361-665X/aae1e1.

16. Chen, L.; Gong X. L,; Jiang W. Q.; Investigation on magnetorheological elastomers based on natural rubber. Journal of Materials Science, 2007, 42: 5483-5489.

17. Feldman, D.; Polymer weathering: photo-oxidation. J. Polym. Environ.; 2002, 10, 163-173. doi: 10.1023/A:1021148205366

18. Masbowski, M.; Miedzianowska, J.; Strzelec, K.; Reinforced, extruded, isotropic magnetic elastomer composites: Fabrication and properties, Adv. Polym. Technol.; 2019, https://doi.org/10.1155/2019/3517430.

19. Li, X.; Bai, T.; Li, Z.; Liu, L.; Inluence of the temperature on the hyper-elastic mechanical behavior of carbon black illed natural rubbers, Mech. Mater. 2016, 95:136-145

20. Zajac, P.; Kaleta, J.; Lewandowski, D.; Isotropic magnetorheological elastomers with thermoplastic matrices: structure, damping properties and testing, Smart Materials and Structures, 2010, 19: 045014 (7 pp.).

21. Akil, H. M.; Cheng, L. W.; Mohd Ishak, Z. A.; Bakar, A. A.; Abd Rahman, M. A.; Water absorption study on pultruded jute fibre reinforced unsaturated polyester composites, Comp. Sci. Technol,; 2009, 69, 1942-1948. doi: 10.1016/j.compscitech.2009.04.014

22. Dhakal, H. N.; Zhang, Z. Y.; Richardson, M. O. W.; Effect of water absorption on the mechanical properties of hemp fibre reinforced unsaturated polyester composites, Comp. Sci. Technol.; 2007, 67, 1674-1683. doi: 10.1016/j.compscitech.2006.06.019

23. Gu, H.; Degradation of glass fibre/polyester composites after ultraviolet radiation. Mater. Des.; 2008, 29, 1476-1479. doi: 10.1016/j.matdes.2007.07.010

24. Jia, Z.; Li, X.; Zhao, Q.; Effect of artificial weathering on surface properties of unsaturated polyester (UP) resin, Mater. Chem. Phys.; 2010, 121, 193-197. doi: 10.1016/j.matchemphys.2010.01.021

25. Chen, L.; Gong, X. L.; Li, W. H.; Microstructures and viscoelastic properties of anisotropic magnetorheological elastomers, Smart Materials and Structures, 2007a, 16: 2645-2650.

26. Kashi, S.; Varley, R.; De Souza, M.; Al-Assai, S.; Di Pietro, A.; de Lavigne, C.; Fox, B.; Mechanical, thermal, and morphological behavior of silicone rubber during accelerated aging, Polym. Plast. Technol. Eng. 2018, 57(16):1687-1696

27. Aziz, S. A. A.; Mazlan, S. A.; Ubaidillah, Mohamad, N.; Choi, S. B.; Aziz, M. A. C.; Johari, M. A. F.; Homma, K.; Thermal aging rheological behavior of magnetorheological elastomers based on silicone rubber, Int. J. Mol. Sci.; 2020, 21(23):9007. doi: 10.3390/ijms21239007.

28. Shaik, M. G.; and Karuppaiyan, V.; effect of ageing on the tracking characteristics of high-temperature vulcanized silicone rubber hybrid composites for high voltage insulation, materials, 2020, 13, doi:10.3390/ma13102242

29. Chakraborty, R.; and Reddy, B. S.; Studies on High-Temperature Vulcanized Silicone Rubber Insulators under Arid Climatic Ageing, IEEE Trans. Dielectr. Electr. Insul.; 2017, 24, 1751-1760.

30. Moon, B.; Lee, J.; Park, S. Seok, C.-S.; Study on the Aging Behavior of Natural Rubber/Butadiene Rubber (NR/BR) Blends Using a Parallel Spring Model, Polymers (Basel).; 2018, 10, 658. https://doi.org/10.3390/polym10060658.

31. Woo, C. S.; Choi, S. S.; Lee, S. B.; Kim, H. S.; Useful lifetime prediction of rubber components using accelerated testing, IEEE T. Reliab.; 2010, 59(1):11-17

32. Wei, H.; Guo, L.; Zheng, J.; Huang, G.; Li, G.; Efect of nanosilica-based immobile antioxidant on thermal oxidative degradation of SBR, RSCAdv.; 2015, 5:62788-62796

33. Liu, J.; Li, X.; Xu, L.; Zhang, P.; Investigation of aging behavior and mechanism of nitrile-butadiene rubber (NBR) in the accelerated thermal aging environment, Polym. Test.; 2016, 54:59-66

34. Ravindran, A.; Kamaraj, M.; Vasanthmurali, N.; Meghavarashini, V.; Balachandran, M.; Nanosilica reinforced EPDM silicone rubber blends: Experimental and theoretical evaluation of mechanical and solvent sorption properties, Materials Today, 2020, https://doi.org/10.1016/j.matpr.2020.09.666

35. Iryani, A.; Masudi, A.; Rozafia, A. I.; Hartanto, D.; Santoso, M.; Nur, H.; Azami, M. S.; Enhanced Removal of Soluble and Insoluble Dyes over Hierarchical Zeolites: Effect of Synthesis Condition, Inorganics, 2020, 8(9), 52; https://doi.org/10.3390/inorganics8090052 
36. Masudi, A.; Jusoh, N. W. W.; Jusoh, R.; Jaafar, N. F.; Jalil, A. A.; Firdaus, A.; Hartanto, D.; Equidistant crystal distortion arrangement of copper doped magnetite for paracetamol degradation and optimization with response surface methodology (RSM), Materials Chemistry and Physics, 2020.

37. Perales-Martinez, I. A.; Palacios-Pineda, L. M.; Lozano-Sanchez, L. M.; Martinez-Romero, O.; PuenteCordova, J.; Elias-Zuniga, A.; Enhancement of a magnetorheological PDMS elastomer with carbonyl iron particles, Polym. Test.; 2016, 57, DOI: 10.1016/j.polymertesting.2016.10.029

38. Palacios-pineda, L. M., Perales-martinez, I. A., Lozano-sanchez, L. M., Mart, O., and Segura-c, E., Magnetorheological Behavior of PDMS Elastomer Reinforced with Iron Micro / Nanoparticles.

39. De Souza Neto, F. N. et al., Particles that slide over the water surface: Synthesis and characterization of iron oxides particles coated with PDMS, with hydrophobic and magnetic properties, Mater. Chem. Phys.; 2015, 162, pp. 100e - 105.

40. Model, C. M. M.; Soria-hern, C. G.; Palacios-pineda, L. M.; El, A.; Investigation of the Effect of Carbonyl Iron Micro-Particles on the Mechanical and Rheological Properties of Isotropic and Anisotropic MREs, Compos. Part B, 2020.

41. Hanoosh, W.S. and Abdelrazaq, E. M.; Polydimethyl siloxane toughened epoxy resins: tensile strength and dynamic mechanical analysis, Malaysian Polymer Journal, 2009, Vol. 4, No.2, p 52-61, 